\title{
Correspondence
}

\section{Thyroid hormones enhance growth and counteract apoptosis in human tenocytes isolated from rotator cuff tendons}

This article has been corrected since Online Publication and a corrigendum has also been published

\author{
F Oliva ${ }^{1}$, AC Berardi ${ }^{2}$, S Misiti ${ }^{3}$, C Verga Falzacappa ${ }^{3}$, A lacone $^{2}$ and $N$ Maffulli*,4,5,6
}

Cell Death and Disease (2013) 4, e705; doi:10.1038/cddis.2013.229; published online 4 July 2013

Subject Category: Experimental Medicine

Dear Editor,

The relationship between thyroid disorders and shoulder pain has been suspected since the late 1920 s. $^{1}$ More recently, such association has been more formally hypothesized, ${ }^{2}$ and thyroid diseases have been linked to idiopathic tendinopathies. ${ }^{3,4}$
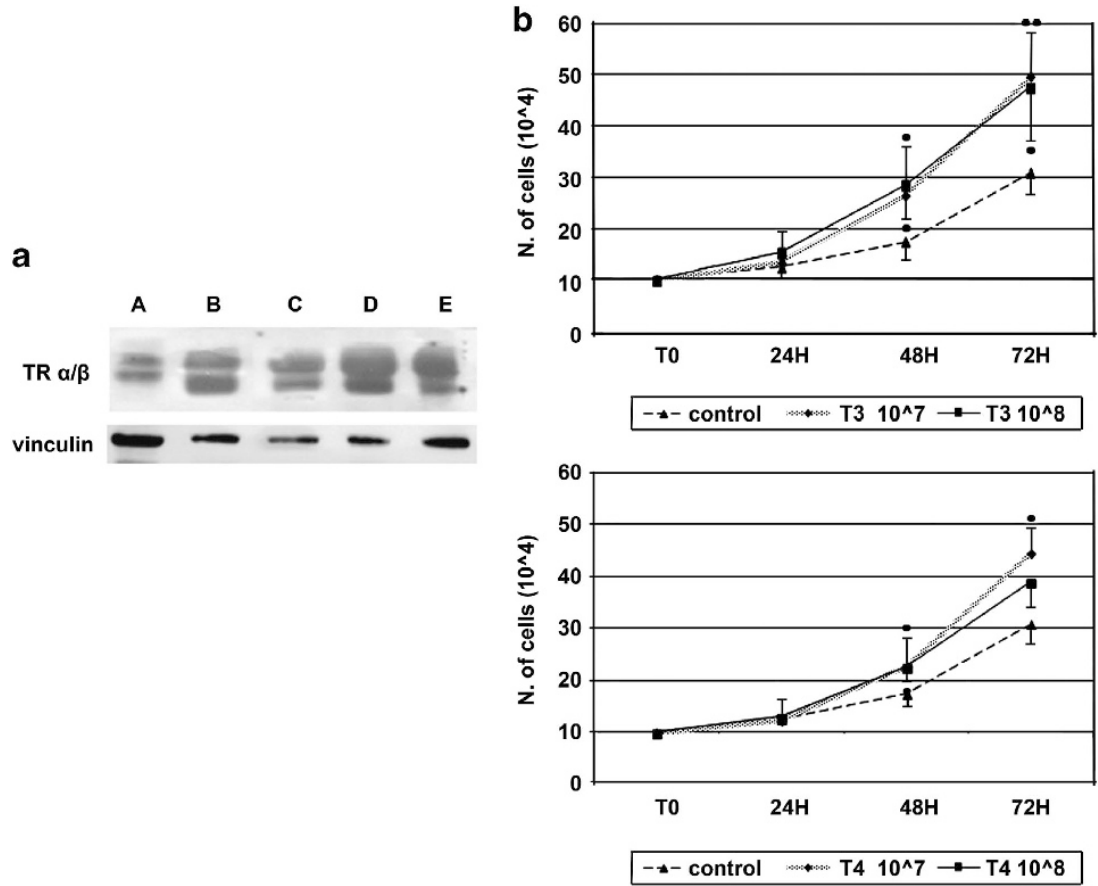

Figure 1 (a) Western blot analysis of TR $\alpha / \beta$ isoforms. A indicates patients with healthy rotator cuff tendons; B-C indicate patients with rotator cuff tears without thyroid disease, D-E represent patients with rotator cuff tears and thyroid disease. The polyclonal antibodies against TRs $\alpha / \beta$ recognize two specific bands at 47 and $55 \mathrm{kDa}$, respectively. At least three different experiments were performed, and a representative one is shown here. (b) Cell growth: tenocyte isolated from healthy tendon were cultured and exposed to different THs concentrations. The graphic shows the effect of $T_{3}$ and $T_{4}$ treatment on cell growth determined by counting trypan blue negative cells; $Y$ axis: cell number, $X$ axis: hours of THs treatment. All the data are presented as mean $\pm S D$, and are the results from five individual experiments. A comparison of the individual treatment was conducted by using one-way ANOVA followed by Turkey post hoc test. Statistical significance in comparison with the corresponding control values indicated by ${ }^{*} P<0.05$ versus control; ${ }^{*} P<0.01$ versus control

\footnotetext{
${ }^{1}$ Department of Orthopaedics and Traumatology, University of Rome "Tor Vergata" School of Medicine, Viale Oxford 81, Rome, Italy; ${ }^{2}$ Laboratory of Stem Cells, Department of Transfusion Medicine, Spirito Santo Hospital, Pescara, Italy; ${ }^{3}$ Department of Experimental Medicine, Endocrinology, Sapienza University of Rome, Rome, Italy; ${ }^{4}$ Faculty of Medicine and Surgery, Department of Musculoskeletal Medicine and Surgery, University of Salerno, Salerno, Italy; ${ }^{5}$ Newham University Hospital, London, UK and ${ }^{6}$ Centre for Sports and Exercise Medicine - Queen Mary, University of London, Barts, UK

*Corresponding author: N Maffulli, Faculty of Medicine and Surgery, Department of Musculoskeletal Medicine and Surgery, University of Salerno, Salerno, Italy or Centre for Sports and Exercise Medicine Queen Mary University of London, Barts, UK or The London School of Medicine and Dentistry, Mile End Hospital, 275 Bancroft Road, London E1 4DG, UK. Tel: + 447989358 279; Fax: + 3906 9417649; E-mail: n.maffulli@qmul.ac.uk
} 
The essential role of thyroid hormones (THs), $\mathrm{T}_{3}$ (triiodothyronine) and $\mathrm{T}_{4}$ (thyroxine), in the development and metabolism of many tissues and organs, both in early and adult life, ${ }^{5}$ is mediated mainly through $\mathrm{T}_{3}$, which regulates gene expression by binding to the TH receptors (TRs) $-\alpha$ and $-\beta$.

Although TRs seem to be ubiquitous, their presence on tendons has not been previously investigated. We therefore evaluated the expression pattern of TR isoforms in three groups of patients: one with rotator cuff tendon tears and thyroid diseases, one with rotator cuff tendon tears without thyroid diseases, and one with healthy rotator cuff tendons. The TR $\alpha$ and TR $\beta$ protein expression level was characterized by western blot analysis (Figure 1a and Supplementary Figure S1a). All healthy and pathologic rotator cuff tendons analyzed expressed high levels of $\mathrm{TR} \alpha / \beta$ nuclear receptor isoforms, indicating that $\mathrm{TR} \alpha / \beta$ expression pattern is not influenced by thyroid diseases.

THs regulate cell proliferation. To further investigate the role played by TRs, we designed in vitro condition that may allow THs to induce proliferation of tenocytes. To this end, tenocytes were isolated from human tendon tissues obtained from five healthy subjects, ${ }^{6}$ and cultured for $72 \mathrm{~h}$ with or without THs. As expected, both $\mathrm{T}_{3}$ and $\mathrm{T}_{4}$ induced cell growth, most effectively at $10^{-7} \mathrm{M}$. The higher increase was obtained by $72 \mathrm{~h}$ of hormone treatment, being $19 \%$ for $\mathrm{T}_{3}$ and $10 \%$ for $\mathrm{T}_{4}$. Tenocytes grew with a doubling time of approximately $49 \mathrm{~h}$. The addition of the THs in the culture medium led to stimulation of cell growth with a reduction of the doubling time. In particular, $\mathrm{T}_{3}$ induced a reduction in doubling time of $27 \%(36 \mathrm{~h})$ and $\mathrm{T}_{4}$ of $19 \%(40 \mathrm{~h}$; Figure $1 \mathrm{~b})$, with the $10^{-7} \mathrm{M}$ dose.

$\mathrm{T}_{3}$ and $\mathrm{T}_{4}$ play an antiapoptotic action. ${ }^{7,8}$ Hence, to verify whether they counteracted apoptosis in isolated tenocytes, cells were plated and serum deprivation was performed for $48 \mathrm{~h}$ to induce apoptosis. Concurrently, tenocytes were exposed or not (control) to $\mathrm{T}_{3}$ or $\mathrm{T}_{4}$. Staining cells simultaneously with Annexin $\mathrm{V}$ and the non-vital dye propidium iodide (PI) allows to identify live (Annexin $\mathrm{V}^{-} \mathrm{PI}^{-}$), apoptotic
(Annexin $\mathrm{V}^{+} \mathrm{PI}^{-}$) and necrotic cells (Annexin $\mathrm{V}^{+} \mathrm{Pl}^{+}$). $\mathrm{T}_{3}$ and $\mathrm{T}_{4}$ caused an increase in vital cells $(83,81$ versus $62 \%)$ and a reduction of apoptotic (5.6, 7.1 versus $18.6 \%)$ cells after $48 \mathrm{~h}$ compared with the control cells (Supplementary Figure S1b).

These results demonstrate that the TR $\alpha / \beta$ nuclear receptor isoforms are present in healthy and pathologic rotator cuff tendons. THs enhance, in vitro, tenocyte growth, and counteract apoptosis in healthy tenocytes isolated from tendon in a dose- and time-dependent manner. Taken together, these results reinforce the concept of a physiological action of THs in the homeostasis of tendons. Much research remains to be performed to clarify the exact role of THs in tendon tissues and their implications in tendon ruptures, tendinopathies and tendon healing. If this association is confirmed, assessment and treatment of patients with tendon conditions may have to be revisited.

\section{Conflict of Interest}

The authors declare no conflict of interest.

Acknowledgements. Part of this work was supported by Associazione ONLUS Laura Bassi.

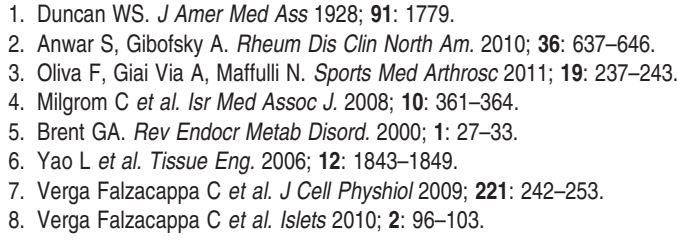

(c) (i) Cell Death and Disease is an open-access journal published by Nature Publishing Group. This work is licensed under a Creative Commons Attribution 3.0 Unported License. To view a copy of this license, visit http://creativecommons.org/ licenses/by/3.0/ 